\title{
Emw1p/YNL313cp is essential for maintenance of the cell wall in Saccharomyces cerevisiae
}

\author{
Correspondence \\ Barry Panaretou \\ barry.panaretou@kcl.ac.uk
}

Received 6 October 2010

Revised 18 January 2011

Accepted 26 January 2011

\author{
Tatjana Sipling, Chao Zhait and Barry Panaretou
}

Pharmaceutical Science Division, King's College London, Franklin-Wilkins Building, 150 Stamford Street, London SE1 9NH, UK

\section{INTRODUCTION}

The tetratricopeptide repeat (TPR) is a structural motif originally identified in yeast as a protein-protein interaction domain (Sikorski et al., 1990). This motif consists of three to 16 tandem repeats of 34 amino acids. Though there is a great deal of degeneracy in the motif, structural analysis indicates a highly conserved 3D structure (D'Andrea \& Regan, 2003). The huge number of protein-protein interactions in yeast revealed by proteome-wide two-hybrid and tandem affinity purification (TAP)-tag datasets indicate the extent to which cell physiology relies on the formation of multi-protein complexes, and it has been estimated that there are five interactions per protein on average (Grigoriev, 2003).

tPresent address: College of Life Science, Hubei University, Wuhan 430062, PR China.

Abbreviations: CFW, Calcofluor White; CWI, cell wall integrity; DAPI, 4',6-diamidino-2-phenylindole; DIC, differential interference contrast; $\mathrm{ER}$, endoplasmic reticulum; GPI, glycosylphosphatidylinositol; MAPK, MAP kinase; TPR, tetratricopeptide repeat.
Twenty-three proteins in Saccharomyces cerevisiae bear TPR domains, of which six are essential (D'Andrea \& Regan, 2003). Three of these six essential proteins are Cdc27, Cdc23 and Cdc16, components of the anaphasepromoting complex (APC/cyclosome), the E3 ubiquitinprotein ligase required for both the metaphase to anaphase transition and subsequent exit from mitosis (Peters, 2006); Tfc4 is a subunit of the RNA polymerase III transcription initiation factor complex (TFIIIC) (Geiduschek \& Kassavetis, 2001), and Prp6 is a component of the U4/ U6-U5 snRNP complex required for pre-mRNA splicing (Legrain et al., 1991). This leaves one essential TPR protein, encoded by YNL313c, for which there is no indication regarding its cellular role. Use of TPRpred (a tool for prediction of TPR repeats from protein sequences) indicates the presence of six TPR domains clustered at the C-terminal end of the protein (Karpenahalli et al., 2007). The remainder of the primary amino acid sequence does not bear recognized motifs from which a function for this $102 \mathrm{kDa}$ protein could be inferred. Interrogation of the protein-protein interaction datasets available for $S$. cerevisiae reveals 21 binding partners involved in numerous 
physiological processes; some of these interactions are bound to be spurious because their subcellular location does not match that of YNL313cp. For instance, there are putative interacting partners located in the mitochondria and the endoplasmic reticulum (ER), yet high-throughput localization of GFP fusions indicates that YNL313cp is found in the nucleus and cytoplasm (Hazbun et al., 2003; Huh et al., 2003).

As we could not infer a possible cellular role for YNL313c via rudimentary bioinformatics analysis or interrogation of the genome/proteome-wide datasets, we generated conditional mutants of the corresponding gene and assessed the phenotypes displayed by these mutants under nonpermissive conditions. Compromising YNL313cp function led to phenotypes that are hallmarks of defects associated with synthesizing or maintaining the cell wall, and we rename the protein Emwlp (essential for maintenance of the cell wall) accordingly. We go on to show that the defect associated with loss of YNL313cp/Emwlp function is not associated with abrogation of the cell wall integrity (CWI) cascade, but is associated with a process that depends on the donor nucleotide sugar UDP-GlcNAc, an essential precursor for three processes, all of which are associated with cell wall homeostasis.

\section{METHODS}

Strains, plasmids and media. Yeast strains used in this study are listed in Table 1. Plasmids are described in the appropriate sections below. Strains were grown in either rich medium (YPD) or synthetic minimal medium (SD), with appropriate supplements for plasmid maintenance (Rose et al., 1990). Deletion of SLT2/MPK1 was performed by replacing this ORF with $h p h$ (conferring resistance to hygromycin B) via one-step gene replacement, as described by Goldstein \& McCusker (1999). Integration at the correct locus was confirmed by PCR using appropriate primers.

Isolation of EMW1 conditional alleles. The heterozygous EMW1/ emw1 $1 \Delta$ diploid Y21131 was obtained from EUROSCARF (Table 1) and transformed with a centromeric URA3 S. cerevisiae/Escherichia coli shuttle vector (pY33T-E) bearing the EMW1 promoter (a fragment $0.45 \mathrm{~kb}$ upstream of the ATG) fused to the EMW1 ORF (a $2.5 \mathrm{~kb}$ fragment), followed by the $A D H 1$ transcriptional terminator (a $0.4 \mathrm{~kb}$ fragment). The EMW1 promoter and ORF from this vector were then subcloned into a centromeric LEU2 S. cerevisiae/E. coli shuttle vector (again bearing the ADH1 terminator), to give pY111TE. Strain Y21131 was transformed with pY33T-E (to give SPD, Table

Table 1. Strains used in this study

\begin{tabular}{|c|c|c|}
\hline Strain & Description & Source \\
\hline Y21131 & 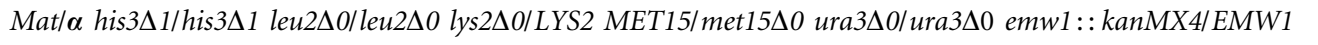 & EUROSCARF* \\
\hline SPD & 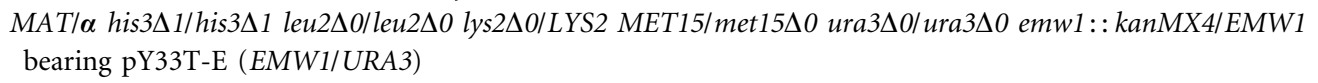 & This study \\
\hline SPE & 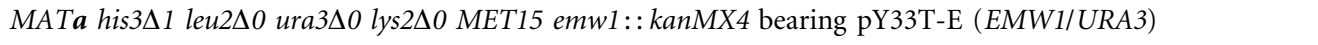 & This study \\
\hline SP0 & 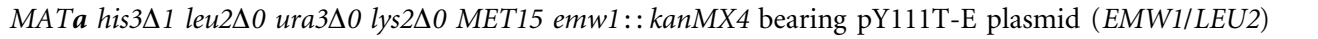 & This study \\
\hline SP1 & SP0 bearing pY111T-e- $1^{\text {ts }}$ plasmid (emw1-1 $\left.1^{t s} / L E U 2\right)$ instead of pY111T-E & This study \\
\hline SP3 & SP0 bearing pY111T-e- $3^{\text {ts }}$ plasmid (emw1-3 $\left.3^{t s} / L E U 2\right)$ instead of pY111T-E & This study \\
\hline SP4 & SP0 bearing pY111T-e- $4^{\text {ts }}$ plasmid $\left(e m w 1-4^{t s} / L E U 2\right)$ instead of pY111T-E & This study \\
\hline SP5 & SP0 bearing pY111T-e- $5^{\text {ts }}$ plasmid (emw $\left.1-5^{t s} / L E U 2\right)$ instead of pY111T-E & This study \\
\hline SP0R & SP0 bearing p2-RLM1 & This study \\
\hline SP1R & SP1 bearing p2-RLM1 & This study \\
\hline SP5R & SP5 bearing p2-RLM1 & This study \\
\hline SP5D & SP5 slt2:: hph bearing pY33T-E (EMW1/URA3) & This study \\
\hline SP0V & SP0 bearing pY195M & This study \\
\hline SP1V & SP1 bearing pY195M & This study \\
\hline SPOE & SP0 bearing pY195M-E & This study \\
\hline SP1E & SP1 bearing pY195M-E & This study \\
\hline $\mathrm{SP} 0 \mathrm{CH} 3$ & SP0 bearing pY195M-C3 & This study \\
\hline $\mathrm{SP} 1 \mathrm{CH} 3$ & SP1 bearing pY195M-C3 & This study \\
\hline SP0GFA1 & SP0 bearing pY195M-GF & This study \\
\hline SP1GFA1 & SP1 bearing pY195M-GF & This study \\
\hline SPG1 & SP0 bearing pM-EMW1-GFP instead of pY111T-E & This study \\
\hline SPG2 & SP0 bearing pE-EMW1-GFP instead of pY111T-E & This study \\
\hline
\end{tabular}

${ }^{\star}$ EUROpean Saccharomyces Cerevisiae ARchive for Functional analysis. 
1). This strain was sporulated, followed by subsequent dissection of tetrads and selection of an $e m w 1 \Delta$ haploid (strain SPE), with viability maintained by the presence of the URA3 vector bearing EMW1 (pY33T-E). Mutations in EMW1 were generated by error-prone amplification of the EMW1 ORF using pY33T-E as template, as described by Fenton et al. (2002). The oligonucleotide primers were designed so that the $5^{\prime}$ primer overlapped the $3^{\prime}$ end of the EMW1 promoter and the $3^{\prime}$ primer overlapped the $5^{\prime}$ end of the $A D H 1$ transcriptional terminator ( $15 \mathrm{bp}$ overlaps in both cases). Strains bearing the mutant emwl alleles were generated by transforming strain SPE with (i) the mutant emw1 PCR products plus (ii) a large linear fragment from pY111T-E lacking the wild-type EMW1. The two fragments recombined with each other in vivo, owing to the 15 bp overlaps between them. The transformants, selected on SD medium lacking both leucine and uracil, possessed the pY33T-E $U R A 3$ vector bearing wild-type $E M W 1$, and a $L E U 2$ vector bearing mutated emwl. More than 4000 transformants were picked and incubated overnight at $25{ }^{\circ} \mathrm{C}$ in 384 -well plates containing SD lacking leucine. The transformants bearing emw $t^{\text {ts }}$ mutants were isolated as described previously ( $\mathrm{Hu}$ et al., 2005). To ensure that these ts phenotypes were attributed to mutated $E M W 1$, plasmids from the strains were recovered, transformed into the SPE strain and rescreened for the ts phenotype. In this way, a series of isogenic emw1 $1 \Delta$ haploid strains were generated, possessing vector-borne copies of wild-type EMW1 (SP0, Table 1) or emw $1^{\text {ts }}$ alleles (SP1-5, Table 1). The coding regions of the emw $1^{t s}$ mutants were sequenced.

Fusions encoding Emwlp-GFP were assembled in pUG23 (kindly provided by J. Hegemann, Heinrich-Heine-Universität, Düsseldorf, Germany), a centromeric HIS3 vector that bears the MET25 promoter, to give pM-EMW1-GFP. The MET25 promoter in pMEMW1-GFP was removed and replaced with the native EMW1 promoter to give pE-EMW1-GFP. The fusions maintained cell

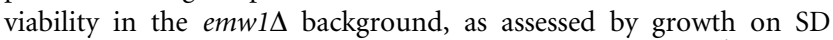
medium without histidine, but containing uracil $\left(50 \mathrm{mg} \mathrm{l}^{-1}\right)$ and 5fluoroorotic acid $(0.1 \%)$, giving strains SPG1 and SPG2 (Table 1).

Sensitivity to cell wall-damaging agents. Exponentially growing cultures in YPD were adjusted to equal cell density $\left(1 \times 10^{7}\right.$ cells $\mathrm{ml}^{-1}$ ) and four successive sixfold serial dilutions were spotted on YPD, or YPD containing the desired concentration of sorbitol, caffeine, Calcofluor White (CFW) or SDS. The plates were incubated at the temperatures specified in the figure legends.

Assessing multicopy suppression by selected ORFs. Candidate genes were overexpressed from the vector pY195M, which is a modification of the episomal URA3 vector YEplac195 (Gietz \& Sugino, 1988). The vector bears the MET25 inducible promoter and the transcription terminator from PGK1. EMW1, CHS3 and GFA1 were amplified from $S$. cerevisiae genomic DNA and ligated into pY195M, to give vectors pY195M-E, pY195M-C3 and pY195M-GF, respectively. The resulting vectors were transformed into the SP0 wild-type strain and the isogenic emw1- ${ }^{t s}$ strain SP1 (Table 1). Expression of the gene was induced by incubation on SD plates lacking methionine.

Assessing the activation state of the MAP kinase (MAPK) CWI pathway. Exponentially growing cultures were either heat-shocked or treated with $10 \mathrm{mM}$ caffeine (as specified in the figure legends). Preparation of total yeast protein extracts was as described previously (Panaretou et al., 1998). Western blots were probed with either (i) anti-(Thr202/Tyr204)-p44/42 MAPK antiserum (New England Biolabs), which specifically recognizes the dually Thr190/Tyr192phosphorylated (activated) Slt2p/Mpk1p in yeast, or (ii) anti-Mpk1/ Slt2 (Santa Cruz). Activity of the Rlmlp transcription factor was assessed using $\mathrm{p} 2-\mathrm{RLM1}$, a YIL117c promoter-LacZ reporter plasmid (Jung et al., 2002).
CFW staining and microscopy. Cells were grown at $25^{\circ} \mathrm{C}$ in YPD to exponential phase and then incubated for $3 \mathrm{~h}$ at the temperatures indicated in the figure legends. Cells were then fixed with formaldehyde and stained with CFW as described by Phelan et al. (2006). Cells were viewed using a Leica DM RXA2 microscope equipped with differential interference contrast (DIC) optics and using an A4 narrowband 4',6-diamidino-2-phenylindole (DAPI) blue filter (340-380 nm excitation wavelength). Images were collected by an ORCA digital camera (Hamamatsu). Emwlp-GFP fusions were localized using a FITC filter.

\section{RESULTS}

\section{Abrogating the function of YNL313cp leads to a catastrophic loss in CWI}

To determine the function of YNL313clEMW1, we generated conditional lethal alleles by PCR-mediated mutagenesis. Five $e m w 1^{t s}$ mutants were isolated, all exhibiting a robust ts phenotype (Fig. 1a). Three mutants were selected for further analysis, based on identifying the mutant with the slowest growth rate, the mutant with the fastest growth rate and a mutant displaying an intermediate growth rate, at permissive temperature. Therefore, the mutants selected were $e m w 1-1^{t s}$,emw1- $5^{t s}$ and $e m w 1-4^{t s}$, which exhibited doubling times of 270,150 and $180 \mathrm{~min}$ at $25^{\circ} \mathrm{C}$, respectively; this was in comparison with the 90 min doubling time displayed by the wild-type. The Emwlp coding regions were sequenced. Most error-prone PCR techniques are not truly random because they yield more transitions than transversions. The method we used, however, removes this bias (Fenton et al., 2002). The amino acid substitutions were: emw1-1 ${ }^{t s}$, L10S, V422G and Y435C; emw1-4 $4^{t s}$ L5S, F282L, K480E, L498P and R896G; and emw1-5 $5^{t s}, \mathrm{~S} 154 \mathrm{P}, \mathrm{I} 454 \mathrm{~T}$, Q511R, Q561L, K704E and S875T.

The emw1- $1^{\text {ts }}$ cells (Fig. 1b) were rounder and larger than wild-type cells at both permissive $\left(25^{\circ} \mathrm{C}\right)$ and nonpermissive $\left(37^{\circ} \mathrm{C}\right)$ temperatures. Increased cell size is typically associated with cell wall mutants (Levin, 2005). The growth defect of emw $t^{\text {ts }}$ cells at non-permissive temperature was alleviated by osmotically stabilizing the medium, and the mutants were sensitive to CFW and SDS, with emw $1-1^{t s}$ exhibiting the most hypersensitive phenotype followed by emw1-4 $4^{t s}$ and $e m w 1-5^{t s}$ (Fig. 2a). The mutants also displayed osmoremedial caffeine sensitivity (Fig. 2b). All of these phenotypes are hallmarks of cells with defects in maintaining integrity of the cell wall (Levin, 2005). CFW blocks chitin polymerization, resulting in a weakened cell wall (Ram \& Klis, 2006). Caffeine blocks cell wall biosynthesis indirectly, and cell wall defects lead to sensitivity to low levels of SDS because a weakened cell wall allows the molecules of the detergent to penetrate more easily (Levin, 2005).

\section{Loss of CWI in the emw $1^{\text {ts }}$ background is not due to a defect in the CWI pathway}

The cell wall defect in emw $1^{\text {ts }}$ mutants may be due to compromised activity of proteins that sense and respond to 

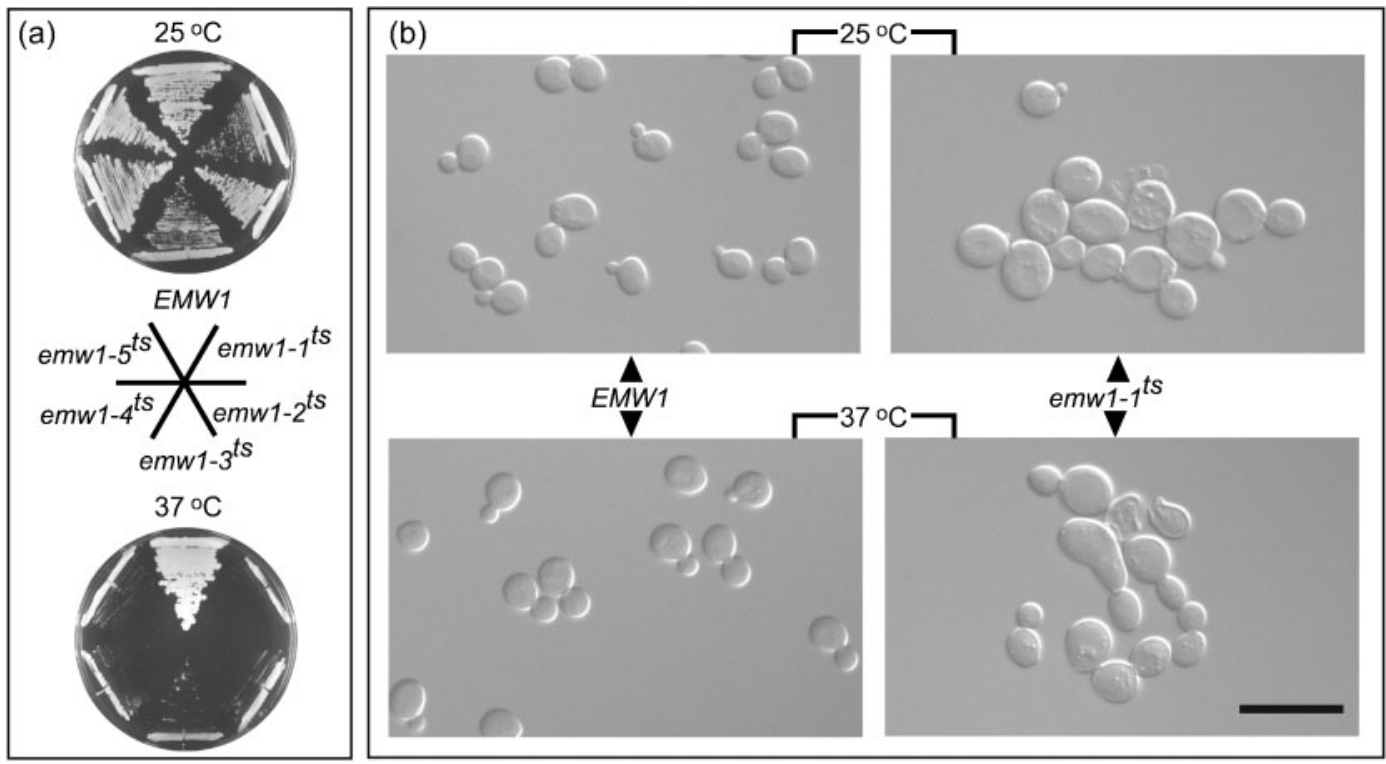

Fig. 1. Phenotype of conditional emw1 mutants. (a) Strains lacking a genomic copy of $E M W 1$, expressing a vector-borne copy of either the wild-type $E M W 1$ or an emw $1^{\text {ts }}$ mutant, were streaked on YPD and incubated for 3 days at 25 or $37^{\circ} \mathrm{C}$. (b) $E M W 1$ wild-type and the emw $1-1^{t s}$ mutant were grown in YPD at $25{ }^{\circ} \mathrm{C}$ to exponential phase. One aliquot of each culture was observed directly, another aliquot was shifted to $37^{\circ} \mathrm{C}$ for $3 \mathrm{~h}$ prior to observation (cells observed at $\times 1000$ magnification; bar, $25 \mu \mathrm{m})$.

cell wall damage. Of all the mechanisms that contribute to regulation of cell wall components, the most important is the CWI MAPK cascade. Cell surface sensors signal to the Rhol GTPase, which activates the cascade, ultimately leading to cell wall remodelling. The pathway ends with phosphorylation of the Slt2p/Mpk1p MAPK, which subsequently phosphorylates and activates the transcription factor Rlm1 (Levin, 2005). This leads to increased transcription of genes encoding proteins that drive cell wall construction, such as CHS3 and FKS2, encoding chitin synthase 3 and a component of the $\beta$-1-3-glucan synthase, respectively (Jung \& Levin, 1999). The MAPK Slt2p/Mpklp kinase is

(a)
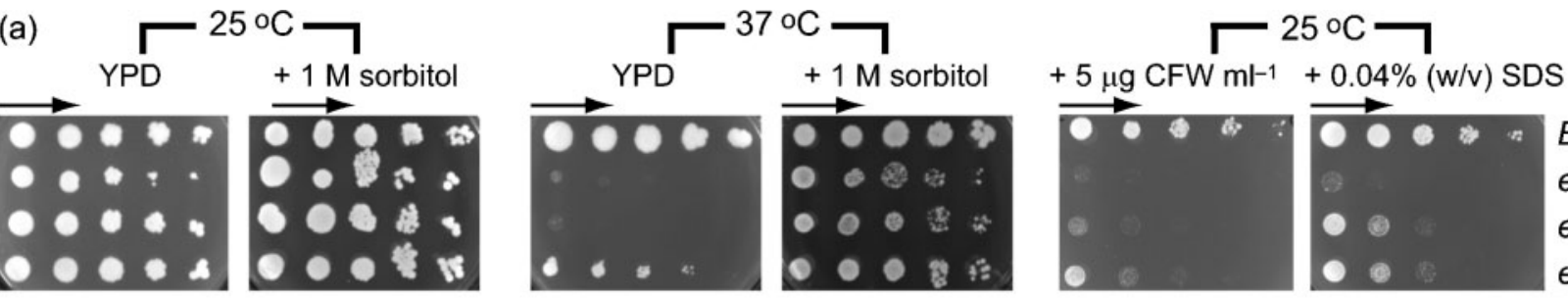

EMW1
emw1-1
emw1-4
emw1-

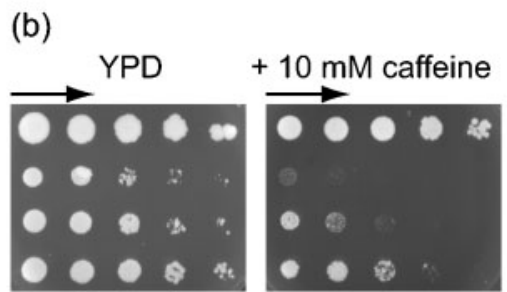

$+10 \mathrm{mM}$ caffeine
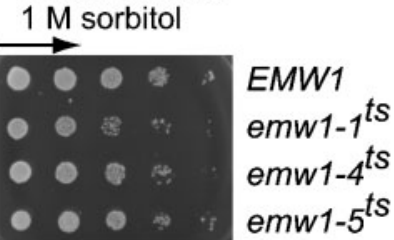

Fig. 2. Loss of Emw1p function leads to defects in CWI. (a) EMW1 wild-type and emw $1^{\text {ts }}$ mutants were grown at $25{ }^{\circ} \mathrm{C}$ to exponential phase and diluted to equal cell density. Sixfold serial dilutions were spotted across YPD (in the direction of the arrow), or YPD containing sorbitol, CFW or SDS, followed by incubation at the temperatures indicated for 3 days. (b) The same cultures were spotted across YPD or YPD containing caffeine or caffeine plus sorbitol, followed by incubation at $30{ }^{\circ} \mathrm{C}$ for 3 days. 
phosphorylated and therefore activated in emw $1^{t s}$ cells at non-permissive temperature (Fig. 3a). The same is true when cells are incubated in the presence of caffeine at permissive temperature (Fig. 3b). Moreover, Slt2p/Mpk1p is constitutively active in the emw $1^{\text {ts }}$ background at permissive temperature in the absence of cell wall destabilizers, whereas we did not detect activated Slt2p/ Mpk1p in wild-type cells (Fig. 3a, b). Not surprisingly, this is reflected by the activation state of the Rlm1 transcription factor under the same conditions (Fig. 3c, d). Accordingly, CWI signalling is not lost when Emwlp function is compromised. Constitutive activation of CWI signalling as the cause of the no-growth phenotype displayed by emw $1^{\text {ts }}$ mutants at non-permissive temperature was ruled out, because the emw1 $1^{\text {ts }} / \operatorname{slt} 2(m p k 1) \Delta$ double mutant was inviable at $25{ }^{\circ} \mathrm{C}$ (Fig. 4). Constitutive activation of Slt2p/Mpklp implies that $e m w 1^{\text {ts }}$ mutants are experiencing cell wall stress. Cell wall mutants, such as those deficient in synthesis of $\beta$-1,3-glucan, $\beta$-1,6glucan, mannosylation (cited in Bulik et al., 2003) and glycosylphosphatidylinositol (GPI) anchors (Sobering et al., 2004), exhibit an increased deposition of chitin in the lateral cell wall as part of a compensatory mechanism. As expected, an increase in chitin deposition was also displayed by emw1 ${ }^{\text {ts }}$ cells (Fig. 5). Moreover, this increase in chitin deposition was observed at permissive as well as non-permissive temperatures (Fig. 5). In cell wall mutants, this is due to upregulation in activity of the chitin synthase Chs3p, which is responsible for making $90 \%$ of cellular chitin (Bulik et al., 2003). Not surprisingly, boosting this compensatory mechanism in the emw1-1 ${ }^{\text {ts }}$ mutant, by ectopic overexpression of $C H S 3$, suppressed the growth defect at non-permissive temperature (Fig. 6a).

\section{Gfa1 is a dosage suppressor of emw1}

Thus far, our data indicate that Emwlp plays an essential role in cell wall homeostasis. This prompted a reexamination of the 21 binding partners of Emwlp identified from the protein-protein interaction datasets available for S. cerevisiae. In particular, we looked for binding partners that are found in the same cellular location as Emwlp. One of these binding partners is Gfalp, a hexosephosphate aminotransferase that converts fructose 6-phosphate to glucosamine 6-phosphate, which is the rate-limiting step in the pathway that synthesizes UDPGlcNAc. Proteome-wide localization studies indicate that Gfalp (Kumar et al., 2002) and Emwlp (Hazbun et al., 2003; Huh et al., 2003) are both found in the cytoplasm. UDP-GlcNAc is the donor nucleotide sugar essential for (a)

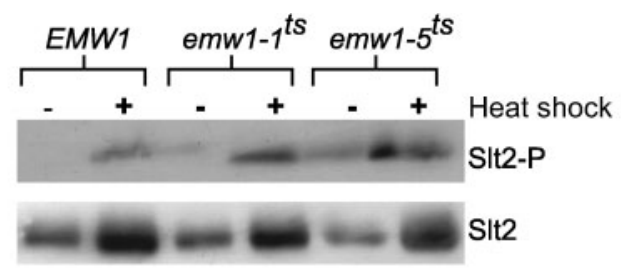

(c)

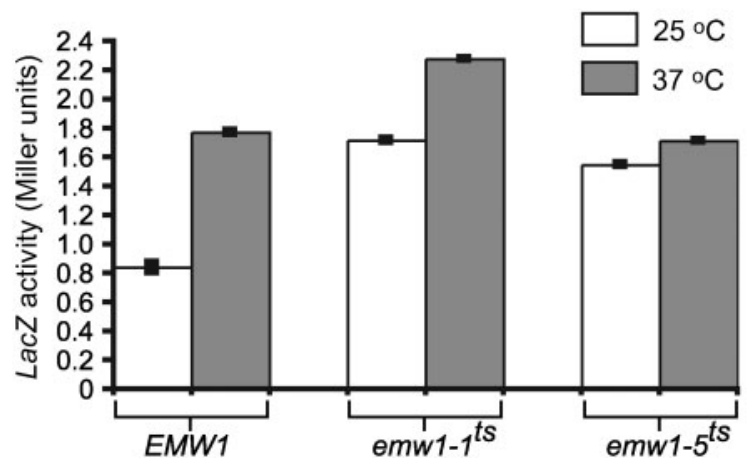

(b)

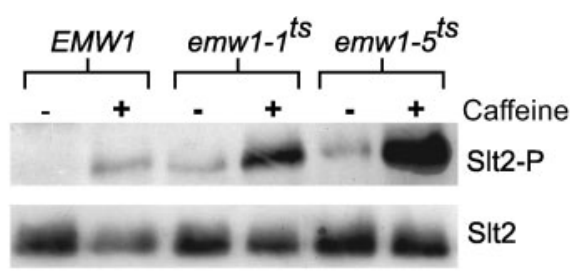

(d)

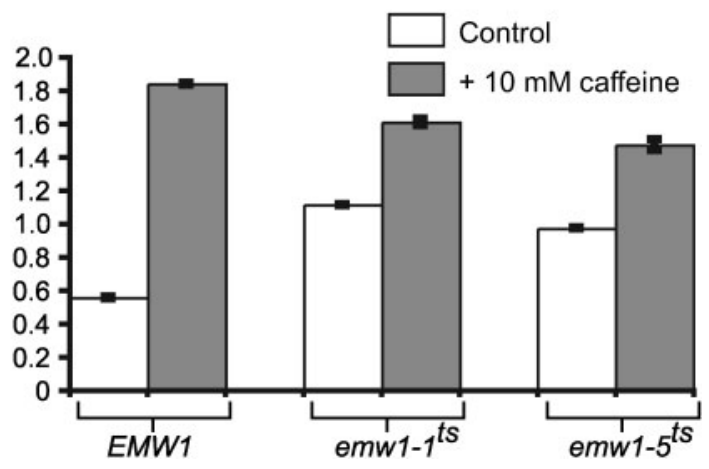

Fig. 3. The MAPK CWI pathway is activated when Emw1p function is lost, and operates normally under conditions that lead to cell wall stress. EMW1 and emw $1^{\text {ts }}$ mutants were grown to exponential phase at $25{ }^{\circ} \mathrm{C}$ and then split into two aliquots, with one aliquot incubated for $1 \mathrm{~h}$ at $25^{\circ} \mathrm{C}$ and the other aliquot subjected to heat shock (incubation for $1 \mathrm{~h}$ at $37^{\circ} \mathrm{C}$ ). The same cultures were grown to exponential phase at $30{ }^{\circ} \mathrm{C}$ and then incubated for $1 \mathrm{~h}$ in the presence or absence of $10 \mathrm{mM}$ caffeine. Cells were harvested and levels of dually phosphorylated Slt2 (indicated as SIt2-P) and total Slt2 were detected on Western blots using appropriate antisera $(\mathrm{a}, \mathrm{b})$. The same strains were transformed with p2-RLM1, a vector bearing a $Y I L 117 \mathrm{c}$ promoter-LacZ fusion. $L a c Z$ activity was assayed after cells were treated in the same way as described above. $Y I L 117 \mathrm{C}-L a c Z$ is a reporter for RIm1-dependent transcription. Data on the $y$ axis are presented as LacZ activity in Miller units; error bars, SEM $(n=3)$. 


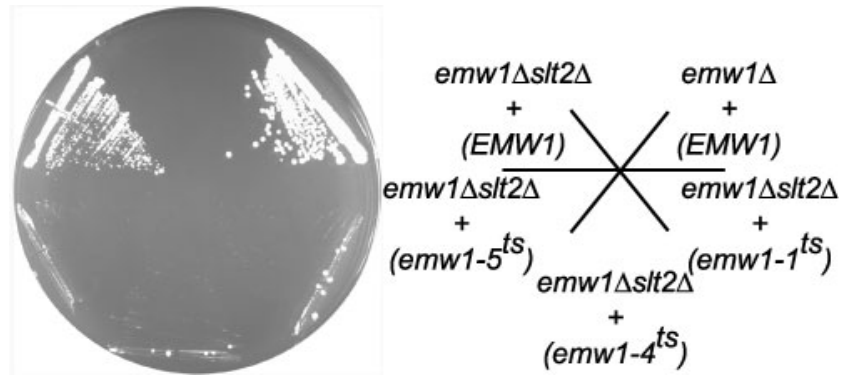

Fig. 4. Synthetic lethality of emw $1 / s / t 2(m p k 1) \Delta$. An emw1 $\Delta s / t 2(m p k 1) \Delta$ strain bearing EMW1 on a URA3 vector was transformed with a $L E U 2$ vector bearing wild-type EMW1 or the mutant emw $1^{\text {ts }}$ alleles indicated in parentheses. Transformants were subsequently incubated on SD with 5-fluoroorotic acid for 3 days at $25{ }^{\circ} \mathrm{C}$. An emw1 $1 \Delta$ strain with intact SLT2(MPK1) bearing both EMW1-URA3 and EMW1-LEU2 vectors was also included (top-right sector). three processes in yeast, all of which are essential for maintenance of the cell wall, namely $\mathrm{N}$-glycosylation, GPIanchor synthesis and chitin synthesis. The interaction of Gfalp with Emwlp is physiologically significant, as overexpression of GFA1 rescued the null-growth phenotype of the emw1-1 $1^{\text {ts }}$ mutant at non-permissive temperature (Fig. $6 \mathrm{~b})$. One interpretation of this result is that Emwlp is required for Gfal function. This could be tested because cells lacking Gfa1p are glucosamine auxotrophs. Exogenous glucosamine bypasses the need for Gfalp, because the amine can enter cells and is subsequently phosphorylated by hexokinases. Indeed GFA1 was originally identified from a screen that selected for glucosamine auxotrophs (Watzele \& Tanner, 1989; Whelan \& Ballou, 1975). However, exogenous glucosamine did not suppress the null-growth phenotype of emw1-1 $1^{t s}$, emw $1-4^{t s}$ or emw1-5 $5^{t s}$ at nonpermissive temperature (not shown). Emwlp is also found in the nucleus, though we were doubtful of localization in this organelle, as examination of the images from the proteome-wide localization studies indicates very weak
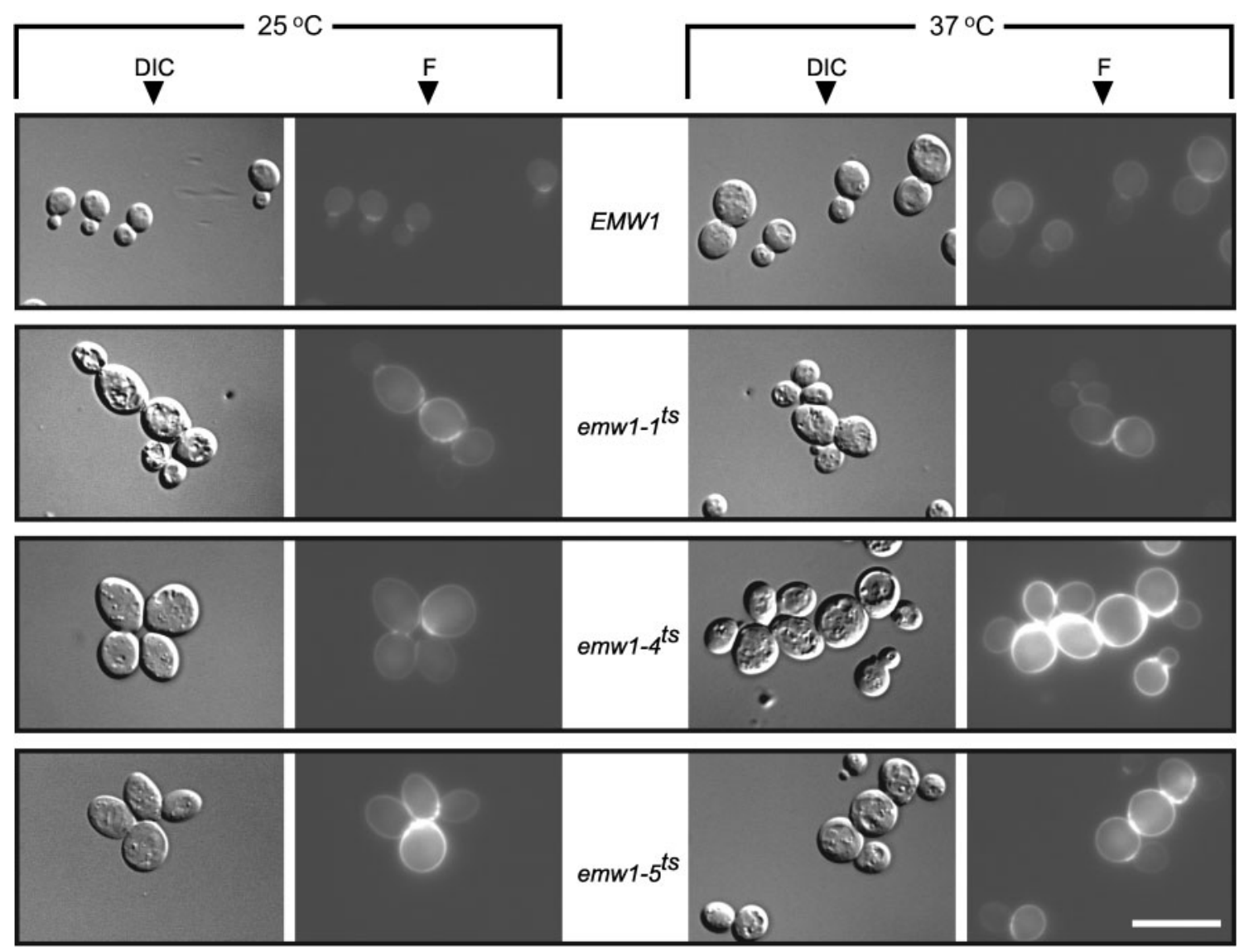

Fig. 5. Excess and aberrant deposition of chitin when Emw1 function is compromised. Wild-type $E M W 1$ and emw ${ }^{\text {ts }}$ mutants were grown to exponential phase at $25{ }^{\circ} \mathrm{C}$. Cultures were divided into two aliquots, one was maintained at $25^{\circ} \mathrm{C}$ and the other was shifted to $37^{\circ} \mathrm{C}$ for $3 \mathrm{~h}$. Cells were fixed, prior to staining with CFW, and then observed under DIC optics and by fluorescence microscopy (F). Images were processed identically in order to preserve relative intensities of fluorescence. Bar, $25 \mu \mathrm{m}$. 
(a)

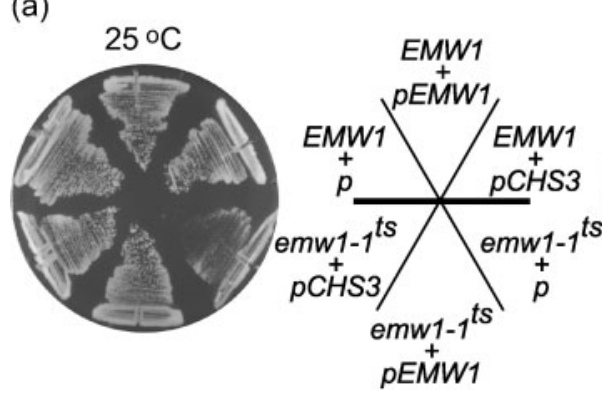

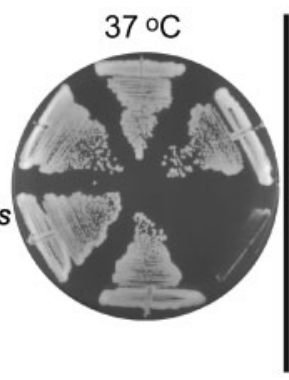

(b)

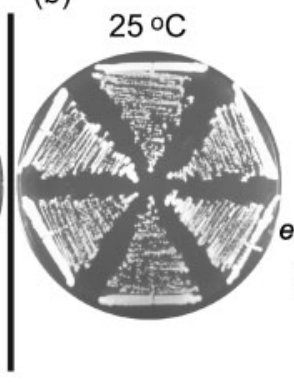

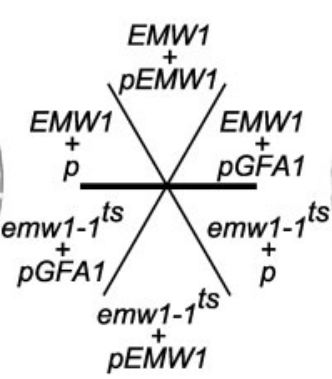

$37{ }^{\circ} \mathrm{C}$

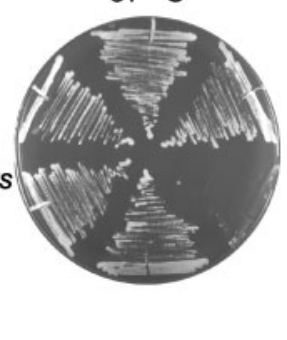

Fig. 6. Dosage suppressors of the emw $1-1^{\text {ts }}$ mutant. Strains lacking a genomic copy of EMW1 but bearing a copy of either the wild-type EMW1 or the emw1-1 ${ }^{t s}$ mutant were transformed with a multicopy URA3 vector (indicated as $p$ ) bearing the ORFs indicated, under the control of the MET25 promoter. Gene expression was induced by inoculating on selective medium lacking methionine followed by incubation for 3 days at 25 or $37^{\circ} \mathrm{C}$.

nuclear fluorescence of Emw1p-GFP fusions. We examined the localization of our own Emwlp-GFP fusion, which retained the essential function of Emwlp as it was able to maintain the viability of emw $1 \Delta$ cells. GFP fluorescence was weak overall, when expression of the fusion gene was driven by the EMW1 promoter, suggesting that native levels of Emw1p are low. Nevertheless, weak fluorescence in both the nucleus and the cytoplasm was detected (Fig. 7a). The nuclear localization was more evident, however, when expression of the fusion gene was driven by the inducible MET25 promoter (Fig. 7b).

\section{DISCUSSION}

The hitherto uncharacterized protein Emwlp does not bear motifs from which a function for this protein could be inferred. However, the protein does bear TPR domains, which are likely to mediate all or some of the interactions with the 21 other proteins identified from the proteinprotein interaction screens applied to S. cerevisiae. Some of these interactions may be artefacts arising from the interaction screening methods. The biologically relevant binding partners may be those that share the most important property of Emwlp, namely that it is required for cell viability. Five of these interacting proteins are essential for viability. These are Rpt5 and Rpn11, which are components of the 26S proteasome (Rubin et al., 1998; Verma et al., 2002); Kar2, a chaperone resident in the ER (Nishikawa et al., 2001); Pta1, a component of the complex that carries out cleavage and polyadenylation of mRNA (Zhao et al., 1999); and Gfa1, which catalyses the ratelimiting step in the pathway that synthesizes UDP-GlcNAc, the donor nucleotide sugar for three processes known to be essential for CWI (Orlean, 1997).

We have provided compelling evidence that Emwlp plays an essential role in maintenance of the cell wall. However, this protein does not play a role in the CWI signalling pathway; indeed, loss of function leads to activation of this cascade. It is unlikely that Emwlp plays a role in the three other pathways that make a comparatively minor contribution to signalling that affects cell wall construction, for the following reasons. (i) Defects in the Rim101 pathway lead to sensitivity to SDS and caffeine (Castrejon et al., 2006); however, unlike $E M W 1$, none of the genes encoding components of this pathway are essential (Peñalva \& Arst, 2002). Also, though rim mutants are sensitive to SDS and caffeine, they are resistant to CFW (de Groot et al., 2001). This is in contrast to emw $1^{\text {ts }}$ mutants, which display a CFW hypersensitive phenotype. (ii) The calcineurin pathway mounts a compensatory response when CWI is compromised. However, (a)

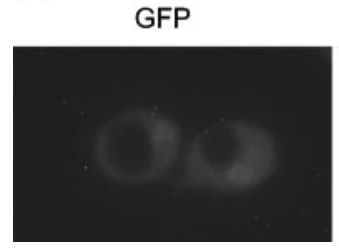

DAPI

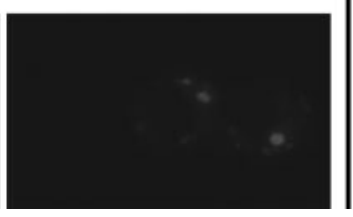

(b)

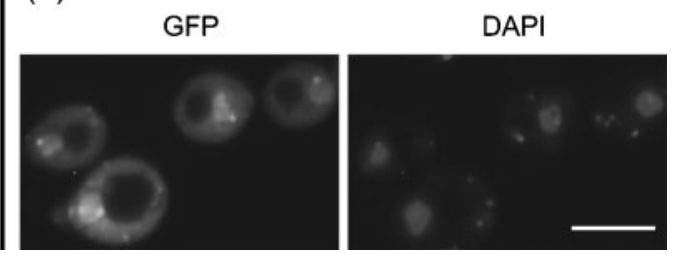

Fig. 7. Distribution of Emw1p. Localization of Emw1p-GFP, via expression of a fusion gene on a centrometic vector under the control of (a) the native EMW1 promoter or (b) the inducible MET25 promoter. The strains were grown to exponential phase in SD medium lacking methionine. Cells were examined by fluorescence microscopy for GFP, with DNA located via DAPI staining. Bar, $25 \mu \mathrm{m}$. 
abrogating this pathway by either deleting all genes encoding the $S$. cerevisiae homologues of calcineurin (Cyert et al., 1991), or deleting the gene encoding the calcineurin-responsive transcription factor Crz1p (Matheos et al., 1997; Stathopoulos \& Cyert, 1997), is not lethal. Furthermore, deletion of the Candida albicans orthologue of CRZ1 renders cells sensitive to SDS, but has no effect on sensitivity to CFW (Santos \& de Larrinoa, 2005). (iii) The high osmolarity (HOG) pathway also influences the plasticity of the cell wall. However, mutants in this pathway are resistant to CFW (García-Rodriguez et al., 2000), in contrast to the hypersensitivity to CFW displayed by emw $1^{\text {ts }}$ mutants.

Activation of the CWI cascade in emw ${ }^{t s}$ mutants indicates that Emw1p plays a more direct role in cell wall biosynthesis. This is plausible, given that $e m w 1^{\text {ts }}$ mutants share a key phenotype with mutants that exhibit defects in the synthesis of the cell wall polymers $\beta$-1,3-glucan and $\beta$ 1,6-glucan, namely an increase in chitin deposition (Bulik et al., 2003). It was not surprising, therefore, to find that GFA1 is a dosage suppressor of the emw $1^{\text {ts }}$ mutant, because Gfalp catalyses the rate-limiting step in the pathway that synthesizes UDP-GlcNAc, the donor nucleotide sugar for three processes known to be essential for CWI, namely $\mathrm{N}$ glycosylation, GPI-anchor synthesis and chitin synthesis. UDP-GlcNAc is the sole donor nucleotide sugar for chitin synthesis. Consequently, if the emw $1^{t s}$ mutation led to loss of Gfalp activity, chitin synthesis would be diminished. Instead, we see a stimulation in synthesis of this polymer. Therefore, the defect in the emw $1^{\text {ts }}$ mutant is associated with a process that depends on UDP-GlcNAc, as opposed to a defect in its synthesis. This is credible because exogenous glucosamine fails to suppress the $e m w 1^{t s}$ growth defect at $37{ }^{\circ} \mathrm{C}$. This leaves the production of GPIanchored proteins and $\mathrm{N}$-glycosylation as the remaining known uses of UDP-GlcNAc. Notably, the defects associated with compromised GPI-anchor biosynthesis are also suppressed by overexpression of GFA1 (Sobering et al., 2004). The only other recorded instance of dosage suppression by GFA1 is reversal of the phenotype caused by a defect in traffic of vesicles from the ER to the Golgi (Morsomme \& Riezman, 2002); the cargo of these vesicles includes GPI-anchored proteins. The step-wise assembly of GPI anchors begins on the cytoplasmic face of the ER, with the transfer of GlcNAc from UDP-GlcNAc to phosphatidylinositol (PtdIns). Following deacylation of the GlcNAc moiety, the resulting glycolipid is flipped to the ER lumen, where the PtdIns is acylated, followed by addition of multiple mannose and phosphoethanolamine moieties, ending with transfer of the GPI anchor to a polypeptide C terminus (Orlean \& Menon, 2007). Emw1p is located in the cytoplasm (Hazbun et al., 2003; Huh et al., 2003), so it could take part in the steps associated with GPI-anchor biosynthesis that take place on the cytoplasmic face of the ER membrane. That GPI anchoring is defective in $e m w 1^{\text {ts }}$ cells is further supported by closer examination of the mutant phenotype. Firstly, emw $1^{t s}$ cells display a cell separation defect, thereby forming aggregates (Figs 1 and 5).
This could be due to limited targeting of the GPI protein Egt2, which plays a role in degradation of the septum (Fujita et al., 2004). Secondly, emw $1^{\text {ts }}$ cells display increased deposition of chitin specifically in the lateral walls of mother cells (Fig. 5); the same is true in mutants lacking the GPI-anchored protein Ecm33, as well as in cells with defects in GPI anchoring itself (Pardo et al., 2004; Sobering et al., 2004). We also note that the GPI assembly pathway shares the UDP-GlcNAc activated sugar precursor with the dolichol pathway for $\mathrm{N}$-glycosylation. $\mathrm{N}$-Linked glycosylation begins on the cytoplasmic face of the ER, with the sequential addition of two GlcNAc moieties and five mannose moieties to the carrier dolichol pyrophosphate. All of these steps are essential. Given its localization, Emwlp may be required for $N$-glycosylation steps on the cytoplasmic face of the ER membrane. It is conceivable that both $\mathrm{N}$-glycosylation and GPI-anchor biosynthesis are defective in $e m w 1^{\text {ts }}$ cells. Ynl313cp/Emw1 may play a role in delivery of UDP-GlcNAc to the cytoplasmic face of the ER. Furthermore, defects in both $N$-glycosylation and GPIanchor assembly lead to defects in the secretory pathway (Copic et al., 2009). Consequently, we would expect a genetic interaction between $E M W 1$ and components of the secretory pathway. In agreement with this is the recent finding that compromising the function of Sec15, which is required for the polarized targeting of vesicles to sites of endocytosis, is synthetically lethal in combination with limiting the expression of EMW1 (Davierwala et al., 2005).

Emwlp is found in all fungi, and orthologues are also found across the eukaryotic lineage. Therefore, its essential function is likely to be associated with a core physiological process. As the protein is localized to the nucleus as well as the cytoplasm, it is possible that it interacts with different proteins in these two locations, these interactions being mediated by the six TPR domains found towards the C terminus of Emwlp. $S$. cerevisiae devotes a significant proportion of metabolic effort to synthesizing and maintaining the cell wall, with over 1000 genes displaying a cell wall phenotype when deleted (Klis et al., 2006). However, only a few of these genes are essential, owing to the redundancy associated with cell wall biosynthesis. For instance, there are three chitin synthases in $S$. cerevisiae (Lenardon et al., 2010). EMW1 joins the select group of essential genes which are indispensable for maintaining the integrity of the cell wall.

\section{ACKNOWLEDGEMENTS}

This work was supported by a PhD studentship to T.S. from the Biotechnology and Biological Sciences Research Council (BBSRC).

\section{REFERENCES}

Bulik, D. A., Olczak, M., Lucero, H. A., Osmond, B. C., Robbins, P. W. \& Specht, C. A. (2003). Chitin synthesis in Saccharomyces cerevisiae in response to supplementation of growth medium with glucosamine and cell wall stress. Eukaryot Cell 2, 886-900. 
Castrejon, F., Gomez, A., Sanz, M., Duran, A. \& Roncero, C. (2006). The RIM101 pathway contributes to yeast cell wall assembly and its function becomes essential in the absence of mitogen-activated protein kinase Slt2p. Eukaryot Cell 5, 507-517.

Copic, A., Dorrington, M., Pagant, S., Barry, J., Lee, M. C., Singh, I., Hartman, J. L., IV \& Miller, E. A. (2009). Genomewide analysis reveals novel pathways affecting endoplasmic reticulum homeostasis, protein modification and quality control. Genetics 182, 757-769.

Cyert, M. S., Kunisawa, R., Kaim, D. \& Thorner, J. (1991). Yeast has homologs (CNA1 and CNA2 gene products) of mammalian calcineurin, a calmodulin-regulated phosphoprotein phosphatase. Proc Natl Acad Sci U S A 88, 7376-7380.

D’Andrea, L. D. \& Regan, L. (2003). TPR proteins: the versatile helix. Trends Biochem Sci 28, 655-662.

Davierwala, A. P., Haynes, J., Li, Z., Brost, R. L., Robinson, M. D., Yu, L., Mnaimneh, S., Ding, H., Zhu, H. \& other authors (2005). The synthetic genetic interaction spectrum of essential genes. Nat Genet 37, 1147-1152.

de Groot, P. W., Ruiz, C., Vázquez de Aldana, C. R., Duenas, E., Cid, V. J., Del Rey, F., Rodríquez-Peña, J. M., Pérez, P., Andel, A. \& other authors (2001). A genomic approach for the identification and classification of genes involved in cell wall formation and its regulation in Saccharomyces cerevisiae. Comp Funct Genomics 2, 124-142.

Fenton, C., Xu, H., Petersen, E. I., Petersen, S. B. \& el-Gewely, M. R. (2002). Random mutagenesis for protein breeding. Methods Mol Biol 182, 231-241.

Fujita, M., Yoko-o, T., Okamoto, M. \& Jigami, Y. (2004). GPI7 involved in glycosylphosphatidylinositol biosynthesis is essential for yeast cell separation. J Biol Chem 279, 51869-51879.

García-Rodriguez, L. J., Durán, A. \& Roncero, C. (2000). Calcofluor antifungal action depends on chitin and a functional high-osmolarity glycerol response (HOG) pathway: evidence for a physiological role of the Saccharomyces cerevisiae HOG pathway under noninducing conditions. J Bacteriol 182, 2428-2437.

Geiduschek, E. P. \& Kassavetis, G. A. (2001). The RNA polymerase III transcription apparatus. J Mol Biol 310, 1-26.

Gietz, R. D. \& Sugino, A. (1988). New yeast-Escherichia coli shuttle vectors constructed with in vitro mutagenized yeast genes lacking sixbase pair restriction sites. Gene 74, 527-534.

Goldstein, A. L. \& McCusker, J. H. (1999). Three new dominant drug resistance cassettes for gene disruption in Saccharomyces cerevisiae. Yeast 15, 1541-1553.

Grigoriev, A. (2003). On the number of protein-protein interactions in the yeast proteome. Nucleic Acids Res 31, 4157-4161.

Hazbun, T. R., Malmström, L., Anderson, S., Graczyk, B. J., Fox, B., Riffle, M., Sundin, B. A., Aranda, J. D., McDonald, W. H. \& Chiu, C.-H. (2003). Assigning function to yeast proteins by integration of technologies. Mol Cell 12, 1353-1365.

Hu, B., Liao, C., Millson, S. H., Mollapour, M., Prodromou, C., Pearl, L. H., Piper, P. W. \& Panaretou, B. (2005). Qri2/Nse4, a component of the essential Smc5/6 DNA repair complex. Mol Microbiol 55, 1735-1750.

Huh, W. K., Falvo, J. V., Gerke, L. C., Carroll, A. S., Howson, R. W., Weissman, J. S. \& O'Shea, E. K. (2003). Global analysis of protein localization in budding yeast. Nature 425, 686-691.

Jung, U. S. \& Levin, D. E. (1999). Genome-wide analysis of gene expression regulated by the yeast cell wall integrity signalling pathway. Mol Microbiol 34, 1049-1057.

Jung, U. S., Sobering, A. K., Romeo, M. J. \& Levin, D. E. (2002). Regulation of the yeast Rlm1 transcription factor by the Mpk1 cell wall integrity MAP kinase. Mol Microbiol 46, 781-789.
Karpenahalli, M. R., Lupas, A. N. \& Söding, J. (2007). TPRpred: a tool for prediction of TPR-, PPR- and SEL1-like repeats from protein sequences. BMC Bioinformatics 8, 2.

Klis, F. M., Boorsma, A. \& De Groot, P. W. (2006). Cell wall construction in Saccharomyces cerevisiae. Yeast 23, 185-202.

Kumar, A., Agarwal, S., Heyman, J. A., Matson, S., Heidtman, M., Piccirillo, S., Umansky, L., Drawid, A., Jansen, R. \& other authors (2002). Subcellular localization of the yeast proteome. Genes Dev 16, 707-719.

Legrain, P., Chapon, C. \& Galisson, F. (1991). Proteins involved in mitosis, RNA synthesis and premRNA splicing share a common repeating motif. Nucleic Acids Res 19, 2509-2510.

Lenardon, M. D., Munro, C. A. \& Gow, N. A. (2010). Chitin synthesis and fungal pathogenesis. Curr Opin Microbiol 13, 416-423.

Levin, D. E. (2005). Cell wall integrity signaling in Saccharomyces cerevisiae. Microbiol Mol Biol Rev 69, 262-291.

Matheos, D. P., Kingsbury, T. J., Ahsan, U. S. \& Cunningham, K. W. (1997). Tcnlp/Crzlp, a calcineurin-dependent transcription factor that differentially regulates gene expression in Saccharomyces cerevisiae. Genes Dev 11, 3445-3458.

Morsomme, P. \& Riezman, H. (2002). The Rab GTPase Yptlp and tethering factors couple protein sorting at the ER to vesicle targeting to the Golgi apparatus. Dev Cell 2, 307-317.

Nishikawa, S. I., Fewell, S. W., Kato, Y., Brodsky, J. L. \& Endo, T. (2001). Molecular chaperones in the yeast endoplasmic reticulum maintain the solubility of proteins for retrotranslocation and degradation. J Cell Biol 153, 1061-1070.

Orlean, P. (1997). Biogenesis of yeast wall and surface components. In Molecular Cellular Biology of the Yeast Saccharomyces cerevisiae, pp. 229-362. Cold Spring Harbor, NY: Cold Spring Harbor Laboratory.

Orlean, P. \& Menon, A. K. (2007). Thematic review series: lipid posttranslational modifications. GPI anchoring of protein in yeast and mammalian cells, or: how we learned to stop worrying and love glycophospholipids. J Lipid Res 48, 993-1011.

Panaretou, B., Prodromou, C., Roe, S. M., O’Brien, R., Ladbury, J. E., Piper, P. W. \& Pearl, L. H. (1998). ATP binding and hydrolysis are essential to the function of the Hsp90 molecular chaperone in vivo. EMBO J 17, 4829-4836.

Pardo, M., Monteoliva, L., Vázquez, P., Martínez, R., Molero, G., Nombela, C. \& Gil, C. (2004). PST1 and ECM33 encode two yeast cell surface GPI proteins important for cell wall integrity. Microbiology 150, 4157-4170.

Peñalva, M. A. \& Arst, H. N., Jr (2002). Regulation of gene expression by ambient $\mathrm{pH}$ in filamentous fungi and yeasts. Microbiol Mol Biol Rev 66, 426-446.

Peters, J. M. (2006). The anaphase promoting complex/cyclosome: a machine designed to destroy. Nat Rev Mol Cell Biol 7, 644-656.

Phelan, J. P., Millson, S. H., Parker, P. J., Piper, P. W. \& Cooke, F. T. (2006). Fablp and AP-1 are required for trafficking of endogenously ubiquitylated cargoes to the vacuole lumen in S. cerevisiae. J Cell Sci 119, 4225-4234.

Ram, A. F. \& Klis, F. M. (2006). Identification of fungal cell wall mutants using susceptibility assays based on Calcofluor white and Congo red. Nat Protoc 1, 2253-2256.

Rose, M. D., Winston, F. \& Hieter, P. (1990). Methods in Yeast Genetics: a Cold Spring Harbor Laboratory Course Manual. Cold Spring Harbor, NY: Cold Spring Harbor Laboratory.

Rubin, D. M., Glickman, M. H., Larsen, C. N., Dhruvakumar, S. \& Finley, D. (1998). Active site mutants in the six regulatory particle ATPases reveal multiple roles for ATP in the proteasome. EMBO J 17, $4909-4919$. 
Santos, M. \& de Larrinoa, I. F. (2005). Functional characterization of the Candida albicans CRZ1 gene encoding a calcineurin-regulated transcription factor. Curr Genet 48, 88-100.

Sikorski, R. S., Boguski, M. S., Goebl, M. \& Hieter, P. (1990). A repeating amino acid motif in CDC23 defines a family of proteins and a new relationship among genes required for mitosis and RNA synthesis. Cell 60, 307-317.

Sobering, A. K., Watanabe, R., Romeo, M. J., Yan, B. C., Specht, C. A., Orlean, P., Riezman, H. \& Levin, D. E. (2004). Yeast Ras regulates the complex that catalyzes the first step in GPI-anchor biosynthesis at the ER. Cell 117, 637-648.

Stathopoulos, A. M. \& Cyert, M. S. (1997). Calcineurin acts through the CRZ1/TCN1-encoded transcription factor to regulate gene expression in yeast. Genes Dev 11, 3432-3444.
Verma, R., Aravind, L., Oania, R., McDonald, W. H., Yates, J. R., III, Koonin, E. V. \& Deshaies, R. J. (2002). Role of Rpn11 metalloprotease in deubiquitination and degradation by the $26 \mathrm{~S}$ proteasome. Science 298, 611-615.

Watzele, G. \& Tanner, W. (1989). Cloning of the glutamine : fructose6-phosphate amidotransferase gene from yeast. Pheromonal regulation of its transcription. J Biol Chem 264, 8753-8758.

Whelan, W. L. \& Ballou, C. E. (1975). Sporulation in D-glucosamine auxotrophs of Saccharomyces cerevisiae: meiosis with defective ascospore wall formation. J Bacteriol 124, 1545-1557.

Zhao, J., Kessler, M., Helmling, S., O’Connor, J. P. \& Moore, C. (1999). Ptal, a component of yeast CF II, is required for both cleavage and poly(A) addition of mRNA precursor. Mol Cell Biol 19, 7733-7740.

Edited by: K. Kuchler 